\title{
Logic Cells as basic structures to add/drop WDM information signals
}

\author{
J.A. Martin-Pereda ${ }^{*}$, A. González-Marcos \\ E.T.S. Ingenieros de Telecomunicación. Universidad Politécnica de Madrid
}

\begin{abstract}
Nowadays. in order to take advantage of fiber optic bandwidth any optical communications system tends to be WDM. The way to extract a channel, characterized by a wavelength, from the optical fiber is to filter the specific wavelength. This gives the systems a low degree of freedom due to the fact of the static character of most of the employed devices. In this paper we will present a different way to extract channels from an optical fiber with WDM transmission. The employed method is based on an Optically Programmable Logic Cells (OPLC) previously published by us, for other applications as a chaotic generator or as basic element for optical computing.

In this paper we will describe the configuration of the OPLC to be employed as a dropping device. It acts as a filter because it will extract the data carried by a concrete wavelength. It does depend, internally, on the wavelength. We will show how the intensity of the signal is able to select the chosen information from the line. It will be also demonstrated that a new idea of redundant information it is the way of selecting the concrete wavelength. As a matter of fact this idea is apparently the only way to use the OPLC as a dropping device. Moreover, based on these concepts, a similar way to route signals to different routes is reported. The basis is the use of photonic switching configurations, namely Batcher or Bayan structures, where the unit switching cells are the above indicated OPLCs.
\end{abstract}

Keywords: Photonic switching. Optical computing. Add-Drop. WDM

\section{INTRODUCTION}

Most of the published works concerning optical bistable devices have been related with some type of optical computing. In the beginning of the eighties. when this effect was initially analyzed. the main objective was obtaining devices with two principal characteristics. namely. high switching speed and low level of power consuming. These objectives were partially obtained but their application to optical computers seemed a very distant goal. The reason was that the potential obtained advantages were insufficient when compared with the results achieved by their electronic counterparts. Although the theoretical maximum switching speed is much higher than the obtained by conventional processors. the complexity of optical architectures makes them very difficult for develop an all-optical computer. A point should be indicated concerning this difficulty. One of the main lines followed to establish the general frame for an optical computer was to develop architectures similar to those employed in electronic computers. This means that most of the processes follow a serial configuration. This philosophy, obvious when employed signals are electrical, is not so obvious when are optical the signals to be used. One of the more important properties of light is its inherent capability to work in a parallel way. This property is the basis for common image processors employing devices as, for example, spatial light modulators.

Although the application of optical bistable devices to optical computing was not in the nineties so brilliant as first proposals indicated some years before, their use in other fields, mainly optical communications, kept them in a priority level. Many configurations in photonic switching employ different types of optical bistability to perform tasks as signals routing and packet switching. Optical computing has been a very good help to develop structures able to work in optical communications.

The introduction of photonic switching has been, in this way, one of main improvements in future optical communications systems. This fact has come together with other very important concept developed in the last years too This concept is wavelength division multiplexing. WDM. A single fiber is now employed to carry several channels with different wavelengths and they go though the same way without any type of mutual interference or crosstalk. Different signals are in the same space and they may perform different type of operations. Although in the same part of space. this

\footnotetext{
${ }^{*}$ Correspondence: Email iamp@tfo.upm.es: E.T.S. Ingenieros de Telecomunicacion. Universidad Politécnica de Madrid, Ciudad Universitaria. 28040 Madrid. Spain.
} 
characteristic is related, in some way, with the parallel properties of light. As a matter of fact. it is possible to call this characteristic as a type of space multiplexing.

A new possible further step in this process may now be proposed. Most of the nowadays switching and routing configurations employ passive structures to process different lambda signals. They are based on several types of structures. for example. Bragg gratings and different types of etalons. In the first case. a demultiplexed signal. with different lambdas going through different paths, is obtained. In the second one. optical frequencies in a certain band are blocked and other frequencies go out of the structure. Almost in any case. operation is passive in the sense that a particular configuration gives rise to a particular result. A possible way to improve this behavior should be to apply concepts employed in optical computing, in general, and in programmable logic, in particular, to introduce a type of active behavior.

Moreover, another way to approach to optical computing it is possible too. As it has been pointed out before. one of the main drawbacks in optical computing is the serial character of most of the employed configurations. The parallel properties of light are not present in any one of the reported architectures. The present WDM systems in optical communication allow a possible way to implement a type of parallel processing. Different signals to be processed at a certain time may go together through an optical fiber in a parallel way. It will be necessary just to separate them at a certain point and perform the desired operation. Signals may no go, as customary, in a serial form. This possibility opens a different form to operate. As it will be shown in this paper. some possibilities may derive from the above indicated possibility.

From these considerations. a new possible form to work in optical communications and in optical computing will be reported in this paper. We will try first to establish some general concepts. most of them based on previous theories. We will develop some principles in order to develop those concepts. Some of these principles have been employed by the authors in previous works. The main emphasis will be given to the way to add/drop different wavelengths and to route signals according to the information contained in their packets as well as their optical frequency.

\section{SOME CONCEPTS OF WDM NETWORK ELEMENTS}

In order to get a close approach to the intended new way to drop a WDM-channel from a fiber optic line. some concepts about conventional optical add/drop elements ${ }^{1}$ will be reported. These basic concepts will help to develop our present proposed approach.
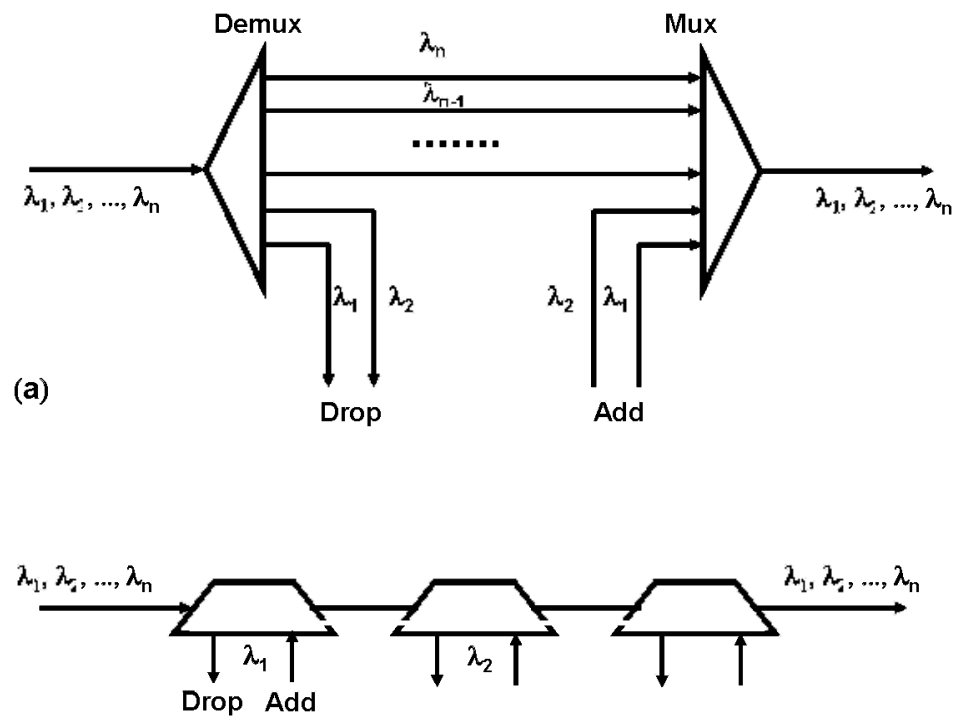

(b)

Fig. 1- Optical Add/Drop architectures. (a) Parallel configuration; (b) Serial structure

Two conventional optical add/drop multiplexers, architectures appear in Fig. 1. These elements provide a costeffective means for handling passthrough traffic in different types of optical networks. Their use is both in long-haul networks and in smaller networks. Fig. 1 shows two different OADM architectures. In Fig. 1.a. a parallel architecture 
appears. All incoming channels are demultiplexed and some of them can be dropped locally and the others are passed through. An arbitrary subset of channels can be dropped and the remaining passed through. So there are no constraints on what channels can be dropped and added. This architecture imposes minimal constrains of planning lightpaths in the network. In addition. the loss is fixed independent of how many channels are dropped and added. This architecture is not very cost-effective for handling a small number of dropped channels because all channels need to be demultiplexed and multiplexed back together. Moreover, wavelength tolerances on the multiplexers and lasers can be fairly stringent. In the serial approach. Fig. 1.b, a single channel is dropped and added from an incoming set of channels. This device is called single-channel OADM (SC-OADM). They can be implemented by using fiber Bragg gratings or dielectric thin-film filters. This architecture complements the previous parallel architecture. Adding and dropping additional channels disrupts existing channels. Therefore it is desirable to plan what set of wavelengths need to get dropped at each location ahead of time to minimize such disruptions. The cost is low if only a small number of channels are to be dropped.

One of the main objectives in OADMs is reconfigurability. Reconfigurability refers to the ability to select the desired wavelengths to be dropped and added on the path. as opposed to having to plan ahead and deploy appropriate equipment. This allows carriers to be flexible when planning the network and allows lightpaths to be set up and taken down dynamically as needed in the network. Architectures in Fig. 1 are not reconfigurable in this sense. The solution to obtain reconfigurable OADMs has been the use of transponders. A transponder, as it is known. adapts the signal coming in from a client of the optical network into a signal suitable for use inside the optical network. In the same way. in the reverse

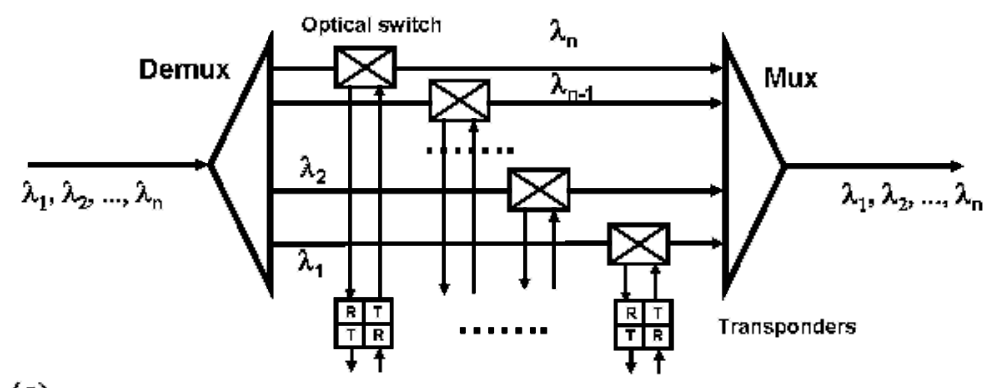

(a)

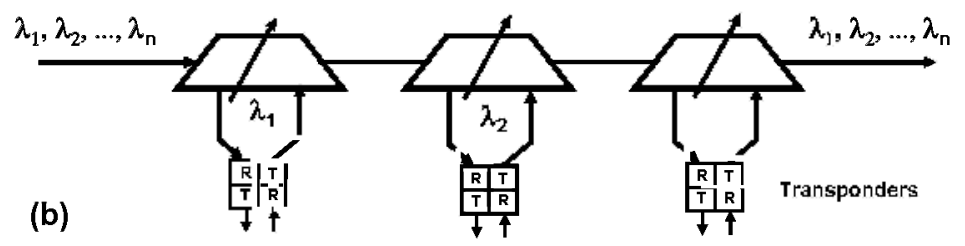

Fig. 2.- Reconfigurable OADM architectures. (a) Parallel. (b) Serial.

direction, it adapts the signal from the optical network into a signal suitable for the client. The architectures in Fig. 1 change to the structures shown in Fig. 2. Fig. 2.a is a partially tunable OADM using a parallel architecture with optical add/drop switches and fixed wavelength transponders. $T$ indicates a transmitter an $\mathrm{R}$ indicates a receiver. Fig. 1.b is a partially tunable OADM using a serial architecture with fixed-wavelength transponders. A problem with these configurations is that they need to deploy the transponders ahead of time so that they are available when needed. This gives rise to two problems. First is expensive to have a transponder deployed and not used while the associated OADM is passing that wavelength through. The second problem is that although the OADMs are reconfigurable, the transponders are not. It is necessary to decide ahead of time the wavelength that will be needed to fix the transponders. In order to avoid these problems is necessary to use tunable transponders.

These problems, although solved with different techniques, indicate that maybe some other type of solution should be looked for. And this is the intention of the present paper. 


\section{A NEW APPROACH TO OPTICAL ADD/DROP MULTIPLEXERS}

Some aspects are needed to be pointed out before we present our solution to above indicated problems. The fist one concerns the way to approach to a different method to drop and add signals. The second one is related with the device and the architecture to be employed. Fig. 2.a showed a wavelength add-drop multiplexer (WADM) attached to a Network Access Station (NAS) through several fiber pairs. This is a natural arrangement when the NAS and WADM are co-located. Optical switches are passive in the sense that they are made in such a way that only the corresponding optical frequency is extracted or added from the line. A separate pair of access fibers is required for each wavelength. The advantage for this configuration is that there is no need for receiver tuning or demultiplexing because only one wavelength reaches each receiver.

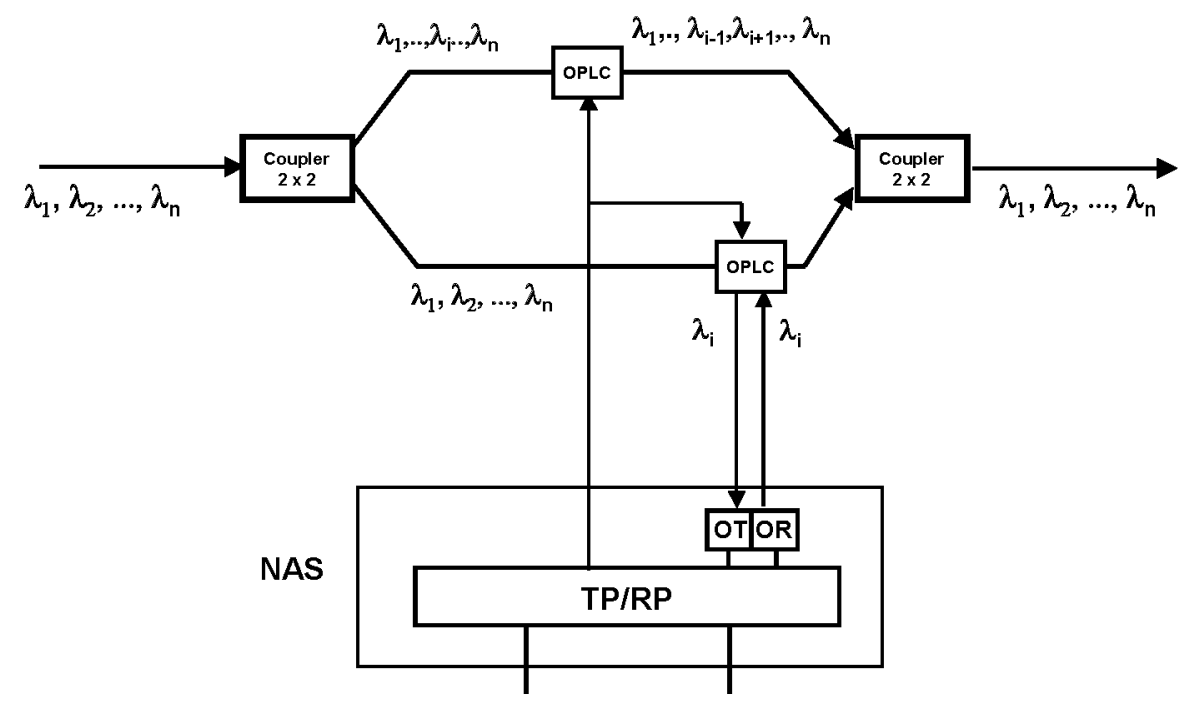

Fig. 3.- Proposed OADM using parallel architecture with optical programmable logic cells and fixed-wavelength transponders. OT and OR correspond to Optical Transmitter and Receptor. NAS is the Network Access Station. TP/RP stands for Transmission/Reception Processor.

The proposed structure is shown in Fig. 3. The main difference with respect to previous configuration is just two pair of fibers are needed as well as a pair of $2 \times 2$ couplers. No mux or demux are needed either. As a matter of fact, incoming signal goes to a simple coupler where is divided into two paths. An OPLC is located in the lower route. According to the signal sent by the TP/RP a certain lambda will be or will not be extracted from there. The OPLC must be able to route the desired optical frequency to the NAS. Other frequencies will be stopped. If design requires that the dropped signal doesn't go through the upper route, a similar OPLC, working in the other fiber, stops its progression in the fiber. Several are the advantages of this structure. The first one coincides with advantages in previous configuration: no need for receiver tuning or demultiplexing. But some others advantages appear. First, extracted optical frequency may be changed according to the order given by the NAS. Each situation determines which one of the possible lambdas will be dropped. The second one is that no wavelength demultiplexing is needed. Simple $1 \times 2$ couplers are needed. Moreover, optical switches are no present in our structure. We need now to indicate the way the OPLC works when frequency dependence is needed. This will be the object of next sections. 


\section{OPTICALLY PROGRAMMABLE LOGIC CELL WITH WAVELENGTH DEPENDENCE}

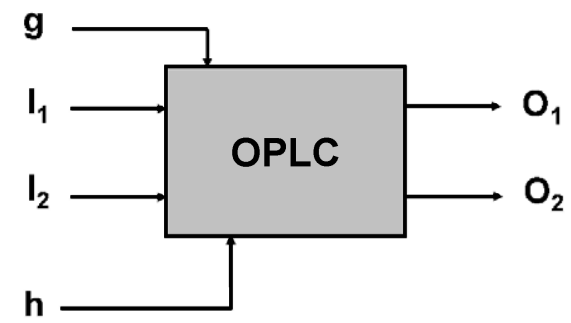

OPLC.

The general behavior of the Optically Programmable Logic Cell has been detailed by us in several previous papers and it will not be repeated here ${ }^{2-4}$. We just indicate its main block structure. It has two signal inputs, $\mathrm{I}_{1}$ and $\mathrm{I}_{2}$, two outputs, $\mathrm{O}_{1}$ and $\mathrm{O}_{2}$, and two control gates, $g$ and $h$. A simple diagram appears in Fig. 4. Two non-linear devices are inside the cell and perform fifteen pairs of Boolean logic functions with input signals. These functions appear at the outputs. In order to achieve the proposed behavior, a device able to perform the required action should be employed. Two are the more important characteristics to be obtained from such a device. The first one is related with the behavior offered with respect to different optical frequencies or wavelengths. The second one concerns to its response with different light intensities impinging on it.

Different types of multi-quantum well, MQW, devices have been proposed and developed in the last years. One of the more employed structures has been the Self-Electrooptic Effect Device, SEED. As it is known, these devices have optical inputs and optical outputs and an electrical current flow within the device. This current

appears because the basic principle of the SEED is to use the current detected in a photodetector to change the electric field across the quantum well region of the modulator. Photodetector and modulator are integrated on the same substrate. The result is a transfer graph, with output light versus input light, showing three main regions of high and low output light. These properties have been employed in several types of structures and applications ${ }^{2-4}$.

Previous applications by us of this device were based on just a simple wavelength going through the structure. Input and output signals were of the same optical frequency. As a consequence, transfer characteristics of the internal non-linear devices were the same for every signal. No modification was needed because no modification was present. But one important property of these devices is their variation with changes in the value of signal wavelength. An example appears

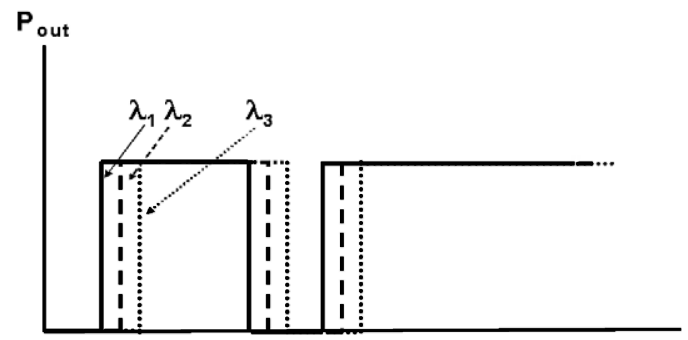

Fig. 6.- Variation with wavelength of $P$ device characteristics in Fig. 4.

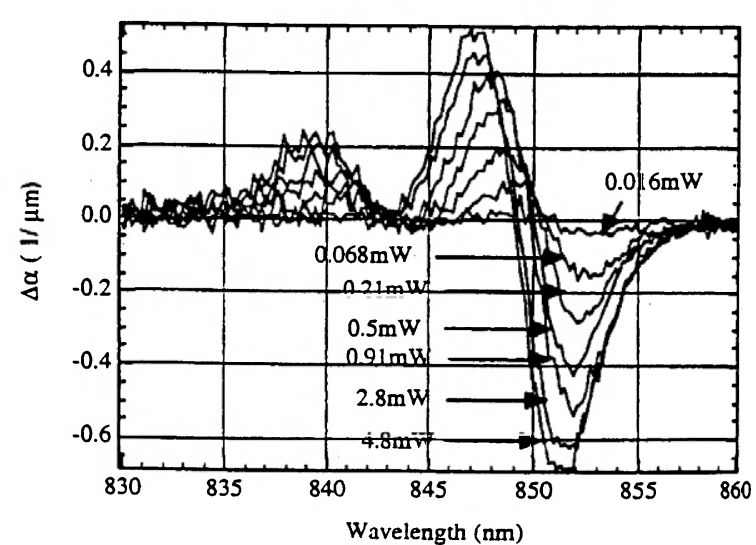

Fig. 5.- Spectrum of absorption change measured in a type II hetero-nipi containing coupled quantum wells ${ }^{5}$.

in Fig. 5 where the variation in absorption versus wavelength is represented for different intensities of the optical signal. A strong non linear effect may be seen with absorption coefficient values changing from a wavelength to another. As it is possible to see, strong variations appear for values between 840 and $855 \mathrm{~nm}$. These changes affect to the general behavior of the device when acting in a similar way to the conventional SEED structure. We have reported this case because variations are larger than with previous conventional structures. As a consequence, the well known output light versus input light representation varies from one frequency to another. 
A problem concerning this behavior is the wavelength range where experiences are reported. Most of the published results in the literature are around a region in the boundary between visible and infrared frequencies. If devices employed in nowadays optical communications systems should be employed. this range is not valid. It should be shifted to higher wavelengths, where second and third windows appear. This fact may be real in the next future. But in order to get a close approach to a point where a more favorable situation will be present, it is convenient to perform some previous analysis of different applications. It is because that we have modeled the new SEED behavior in the way shown in Fig. 6. It is the conventional transfer curve but with the indication of changes with respect to wavelength. This new transfer curve will employed by us in the next approaches to this topic. A similar behavior, but simpler because is just "on-off", is obtained for the Q device of the OPLC structure.

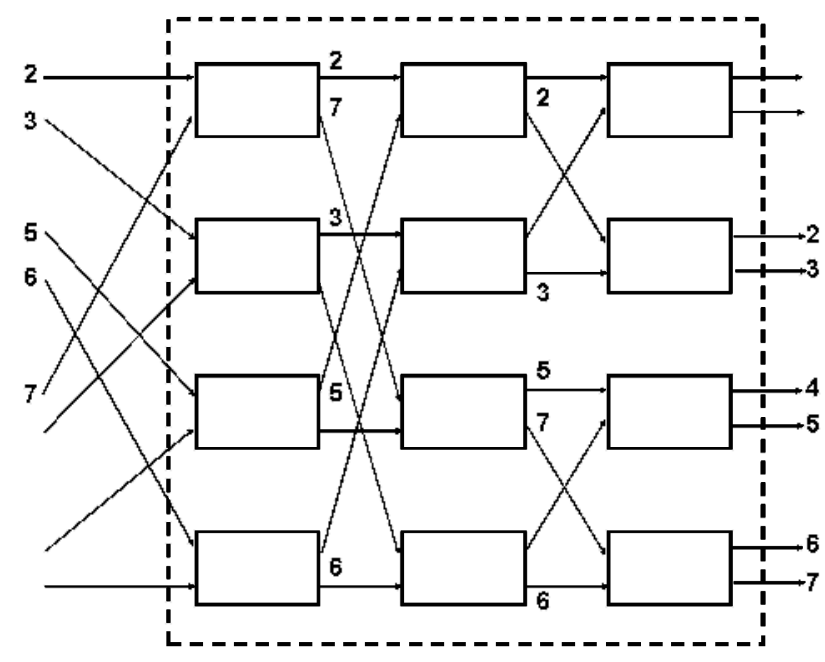

Fig. 7.- A simplified 8 × 8 Banyan network.

\section{SWITCHING ARCHITECTURES WITH OPLCs}

This section discusses some possible switching architectures that use OPLCs as basic units. We will give special emphasis to the case where self-routing is achieved. namely to structures of the Batcher or Banyan type. As it will be shown. the possible application of OPLCs to switching structures gives the possibility to avoid some possible switching conflicts or internal blocking at a certain stage. We will first summarize the properties of conventional Batcher sorting and Banyan routing networks. The final part of this section will propose the new configuration with Optically Programmable Logic Cells as well as its possible application to add-drops.

\subsection{A panoramic of some switching networks: self-routing}

The concept of self-routing is attractive in packet switching where routing is performed at each switching stage according to the destination address of an input packet. In this case must be an address decoding logic at each crosspoint. From this arrangement, a separate switching control to configure the switching fabric is unnecessary. In practice, an input packet carries its routing information through either its virtual circuit identifier (in virtual circuit) or its destination node address (in datagram). When the packet is routed through the switch. one bit at a time of its p-bit address is used for switching at a stage.

A Banyan routing network is shown in Fig. 7. If $\left(\mathrm{n}_{1}, \mathrm{~m}_{2}, \mathrm{~m}_{3}\right)$ is the triple binary output port address of an incoming packet, bit $\mathrm{m}_{\mathrm{l}}$ will be used for routing at stage i. Otherwise, it will be routed to the upper port. For example, if an incoming packet at input port 3 has an output address 6 (both in the range from 0 to 7 ) or (110), it will be routed down in the first stage, stay down in the second stage. and then go up in the last

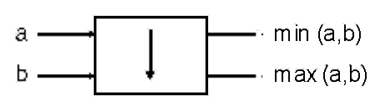

(a)

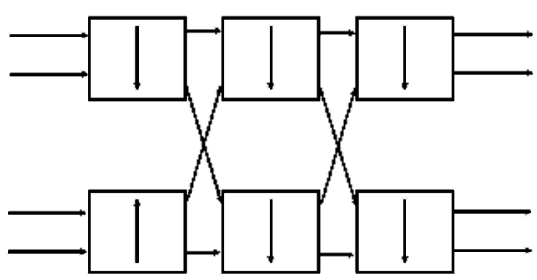

(b)

Fig. 8.- Basic comparison block in a Batcher Network (a) and definition and a 4 x 4 Batcher switch (b). 
stage. A possible problem appears if, for example. another incoming packet at input port 2 that has an output port address 5 (101), it needs to be routed down at the same first stage switch than the previous packet. Therefore, there is a switching conflict, or an internal blocking, at the first stage.

A way to solve this problem is to locate previously a Batcher sorting network. The basic definition to construct it appears in Fig 8. The Batchet network can sort input packets according to their output port addresses in an ascending order. A comparison block compares the inputs coining to its two input ports. According to arrow sense. the input with a maximum value appears at the output where the arrow indicates. Minimum value goes out through the other output. A $8 \times 8$ Batcher appears in Fig. 9. In its first stage two half-size Batcher networks sort inputs into two monotonic subsequences: one increasing and one decreasing. The second stage then merges the two subsequences into one single ascending sequence. According to the merging algorithm by Batcher ${ }^{6}$. corresponding elements in the two subsequences are formed into pairs and compared. Those that are larger are grouped into one new subsequence. and those that are smaller are grouped into another. Each of two subsequences is then split into half for similar comparisons. This process repeats until each of the final subsequences has only one element. The final outcome is a sorted sequence.

The result to combine a sorting network and a self-routing network is a configuration with zero internal blocking. The network of Fig. 10 is an example and it will be the structure to be adopted by us.

\subsection{Implementation of Batcher- Banyan switching structures with Optically Programmable Logic Cells}

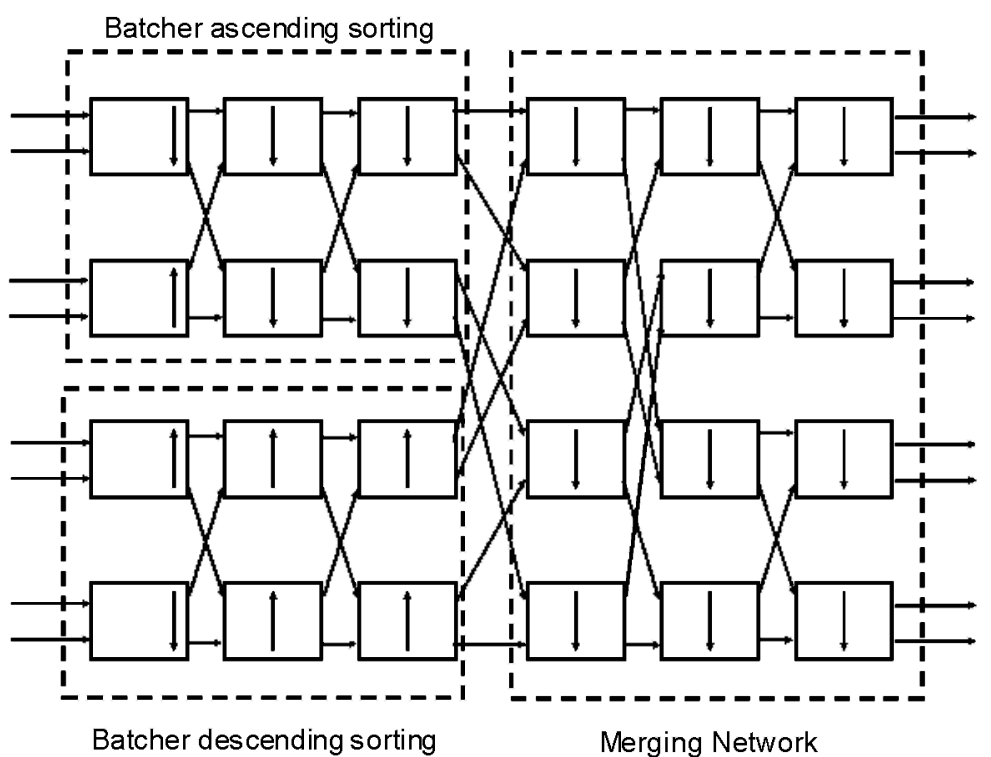

Fig. 9.- An 8 x 8 Batcher network

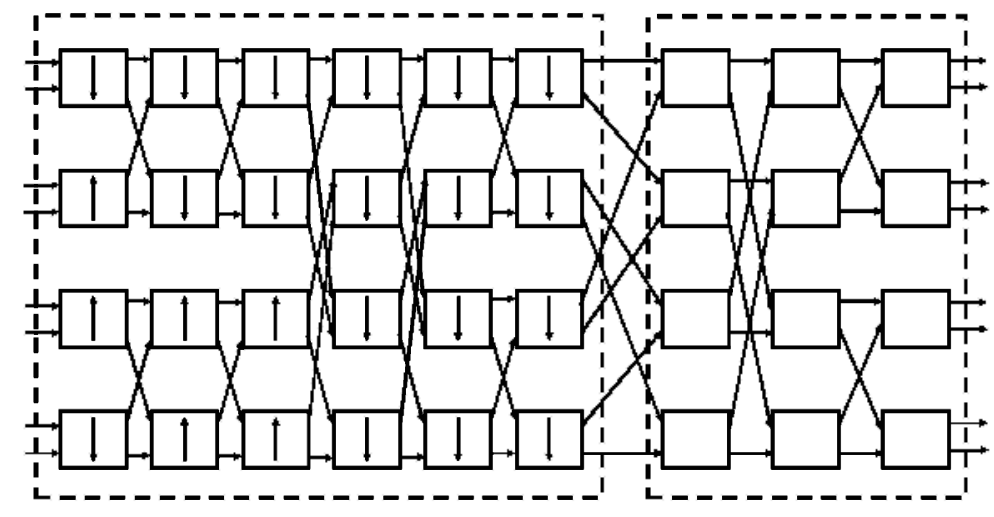

Fig. 10.- An 8 × 8 Batcher - Banyan structure.

In order to construct a switching structure able to route optical packets, a Batcher-Banyan architecture can be arranged with OPLCs as basic units. The general structure is the same one shown in the previous paragraph. We will restrict here to the main points related with the behavior of OPLCs when this application is intended.

The first step will be to determine the way to obtain the behavior indicated in Fig. 8.a. The principal aspect to be considered is the way a bit " 1 " will appear at the port where the arrow indicates, when a bit " 0 " goes through the other input 


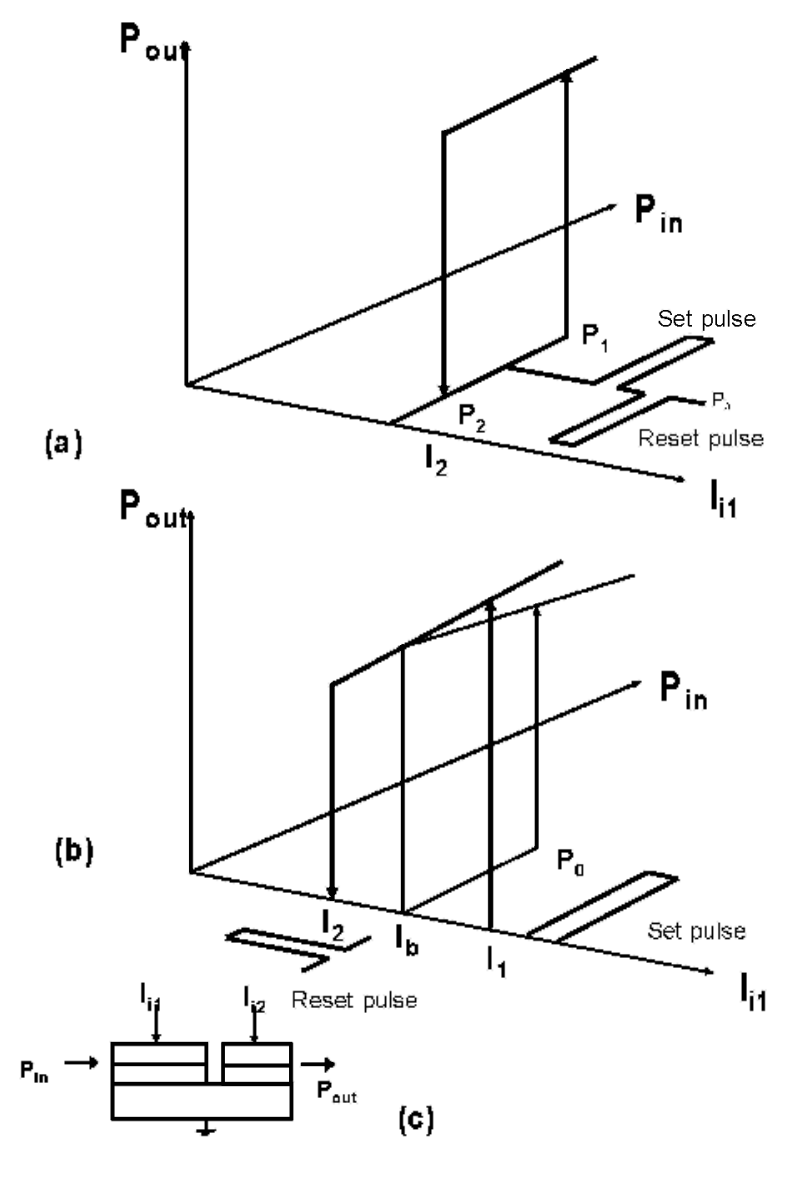

Fig. 11.- (a) (b) Bistable laser diode characteristics

(c) Laser diode structure. port. Input ports where " 1 " and " 0 " bits were going into the cell do not change the result of the operation. If two " 0 "s or two "1"s go to input gates. output gates give rise to the same inputs. This results indicate that the operation performed inside the cell is an OR. Some other logic operations may give the same result but we consider just this one

To perform above indicated operation in the cell. and according to the results reported in previous papers by us ${ }^{2-4}$, it is necessary to control the behavior of the cell by the adequate signal given to the control gate (Fig. 4). As we have indicated previously, this situation corresponds to apply a certain signal with the needed level. This level has to come from the initial bit in the packet. Moreover. this bit has to maintain the same logic function in the OPLC during the time the remaining of the packet last.

The way to achieve the above result is by a bistable laser diode ${ }^{7}$. This bistable laser operates as an optical flip-flop which can be set and reset by optical signals. These signals correspond to the initial bit of the packet. Fig. 11 shows its hysteresis characteristics as the relation among an optical input power $\mathrm{P}_{\mathrm{In}}$, output power $\mathrm{P}_{\text {out }}$ and injection current $\mathrm{I}_{\mathrm{il}}$. This bistable laser diode can operate as an optical memory by two different methods. Fig. 11.a corresponds to a situation where an injection current $I_{i 1}$ is set smaller than threshold current $I_{2}$. The laser reaches beyond a $\mathrm{P}_{1}$ threshold power and laser oscillation stops when optical input power Pin decreases below a $\mathrm{P}_{2}$ threshold power. Therefore, the bistable laser diode has two stable states $\mathrm{E}$ and $\mathrm{G}$ and can retain binary optical information as long as bias input power is remaining on $\mathrm{P}_{\mathrm{b}}$. In the indicated in Fig. 11.b the bistable laser begins oscillation without providing optical input power, when injection current $\mathrm{I}_{\mathrm{i} 1}$ reaches beyond an $\mathrm{I}_{1}$ threshold current. Laser oscillation stops when injection current $\mathrm{I}_{11}$ decreases below an $\mathrm{I}_{2}$ threshold current. This structure and these behaviors allow us to perform the desired function.

The initial bit, corresponding to the packet to be addressed, brings the laser to the upper state and its signal goes to one of the control gates of the OPLC. This signal, as it may be seen in the working diagram of the OPLC. brings it to the state where an OR function is performed. With this function. the output through upper gate will be the remaining bits in the packet. On the contrary, if initial pulse in the packet is a " 0 ", output will go through the lower gate. A simple scheme of this configuration appears in Fig. 12.a. Two possible cases are indicated. Packet A has a bit " 1 " as first digit. Packet B has a " 0 ". As it may be seen in Fig. 12.b, this bit. going through the bistable laser diode configuration, send the corresponding order to one of the OPLC control gates. The following bits in the packet, because the bistable characteristics of the laser, go through the path determined by this first bit. Bistability allows maintaining the same situation during the packet time. After this time. laser diode goes back to the first state, under threshold. Next packet will repeat the same operation.

An advantage of this configuration is that a more versatile behavior may be allowed. As it was pointed out before. if through the second gate of the OPLC goes another signal with an address whose initial bit is a " 1 " too. blocking appears. In the present situations, and according to Fig. 6. two different signals may go through the same path when their wavelengths are different. A possible solution is to convert it before going into the OPLC by a wavelength converter, for instance, a SOA. This allows both signals go through similar paths and have a similar behavior.

Moreover, a Batcher structure may be located before the proposed Banyan. A method, similar to the above reported. can be employed. In this case, a new architecture needs to be present. This happens because is not a " 1 " and a " 0 " the bits that have to be identified. Now it is necessary to determine which one of the two number going into the cell is larger in order to send it to the direction indicated by the arrow. The basis of the operation is now the comparison between two 
numbers. Several are the possibilities. The main difference with respect to the previous operation is the signals analysis

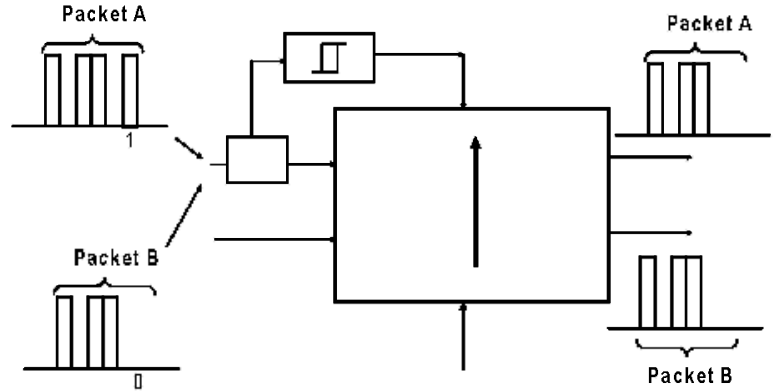

(a)

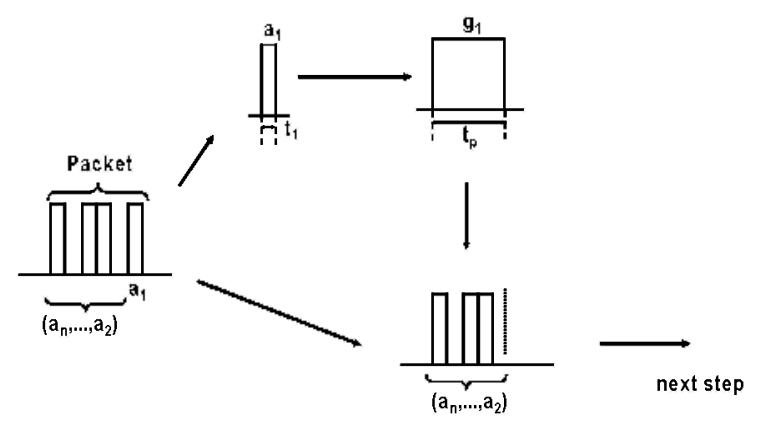

(b)

Fig. 12.- Operational characteristics of basic mechanism to a route packet through the OPLC.

performed before they go through the OPLC. Once it is determined the output gate. a similar mechanism than before is applied, namely, a signal given by a bistable laser diode controls the output port. Differences are in the previous step. A possible solution is to transfer the value of each one of the two arriving numbers to an optical signal with a level proportional to its value. These two levels have to be compared. And the straightforward solution is to set one of then as control signal for an OPLC and allow the other one to pass through the cell. According to their relative values, second signal will pass the cell or it will be stopped.

\section{CONCLUSIONS}

The work reported in this paper is just a simple presentation of some of the possibilities obtained when devices employed in Optical Computing are used in Optical Communications. Moreover, we have shown how concepts employed in Optical Communications may be transferred to Optical Computing. The reported applications to optical add-drops and wavelength switching are a proof of these proposals. Moreover, some other configurations may be implemented when the concepts employed in optical switching should be introduced in the reported add-drops. At the same time, some more details about the internal behavior of the needed devices and structures have to be detailed.

\section{ACKNOWLEDGMENTS}

This work was partly supported by CICYT "Comision Interministerial de Ciencia y Tecnologia". grant TIC99-1131 and CAM "Comunidad Autonoma de Madrid", grant 07T/0037/2000.

\section{REFERENCES}

1. See for example. R. Ramaswami and K. N. Sivarajan. "Optical Networks. A practical Perspective". Morgan Kaufman. San Francisco (2002)

2. A. Gonzalez-Marcos and J.A. Martin-Pereda. "Digital Chaotic Output from an Optically-Processing Element". Optical Engineering. 35. 525-535 (1996)

3. A. González-Marcos and J.A. Martin-Pereda. "Analysis of irregular behaviour on an optical computing logic cell". Optics \& Laser Technology, 32, 457 - 466 (2000)

4. A. González-Marcos and J.A. Martin-Pereda. "Method to analyze the influence of hysteresis in optical arithmetic units". Optical Engineering, 40, 2371-2385 (2001)

5. E. Garmire, "Resonant Optical Nonlinearities in Semiconductors". IEEE Journal of Selected Topics in Quantum Electronics, 6, 1094-1110 (2000)

6. K.E. Batcher, "Sorting Networks and Their Applications", Am. Fed. of Information Processing, 32, $307-314$ (1968) 
7. S. Suzuki, T. Terakado, K. Komatsu, K. Nagashima, A. Suzuki and M. Kondo, "An Experiment on High-Speed Optical Time-Division Switching", IEEE Journal of Lightwave Tecnology, LT-4, 894-899 (1986) 